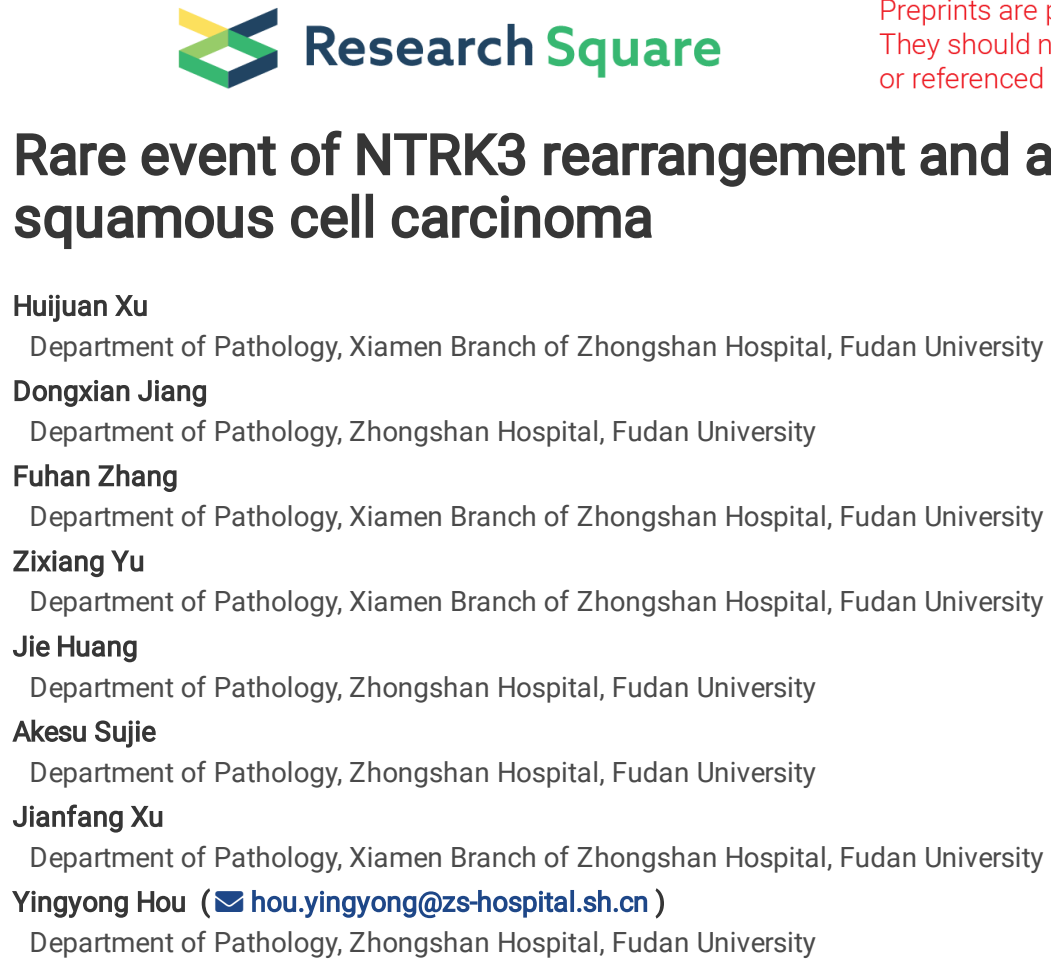

\title{
Rare event of NTRK3 rearrangement and amplification in esophageal squamous cell carcinoma
}




\section{Abstract}

Background: TRK tyrosine kinase inhibitors (larotrectinib or entrectinib) can cause an unknown histological response in NTRK fusion-positive cancer patients,and it has been approved by the US Food and Drug Administration. The neurotrophic tyrosine kinase receptor (NTRK) 3 gene rearrangements is a target of tyrosine kinase inhibitors. We attempted to assess the correlation of NTRK3 gene rearrangement and gene copy number changes with ESCC prognosis.

Methods: The tissue microarray of 478 cases was detected by fluorescence in situ hybridization (FISH) assay.Kaplan-Meier survival and Cox regression analysis were performed to evaluate the prognostic significance of NTRK3 rearrangement and amplification in esophageal squamous cell carcinoma.

Results: This study show that both NTRK3 gene rearrangement and copy number variation are rare events in ESCC. Gene rearrangement and copy number changes have little influence on the prognosis. Except when gene copy number was greater than or equal to 4, patients with stage I+ll showed a trend of poor prognosis $(P=0.120 ; O S, P=0.095)$.

Conclusion: NTRK3 gene rearrangement and gene copy number variation are rare in esophageal cancer, and gene rearrangement does not affect prognosis, while the number of gene copies was 4 as the threshold, there was a tendency of poor prognosis.

\section{Introduction}

ESCC is the fourth most common cause of cancer-related death and one of the most common malignant diseases in the world, especially in China[1, 2]. Esophageal cancer accounts for $70 \%$ of global esophageal cancer in China, of which $95 \%$ is esophageal squamous cell carcinoma (ESCC) [3]. Despite significant improvements in treatment levels in recent years, ESCC is still one of the leading causes of cancer deaths with a 5-year survival rate of less than $20 \%[4]$. It is important to understand prognostic factors, which can help to select more appropriate surgical methods and thus improve the survival of these patients.

Advances in molecular biology have made it possible to detect the molecular changes in human tumors. Study of molecular prognostic markers is one of the hotspots of current research. For example, identifying specific operable oncogenic abnormalities, such as gene activation point mutations, insertions, deletions, and amplifications or rearrangements[5]. Therefore, clarifying the genomic abnormality of esophageal squamous cell carcinoma and elucidating the molecular mechanism of its occurrence and development can guide the development of effective targeted therapy, which is an urgent need for clinicians to improve the survival rate of esophageal squamous cell carcinoma.

Recently, mutations or rearrangements of the troponin receptor kinase (TRK) receptor tyrosine kinase family have been found to be a carcinogenic mechanism[6],and this rearrangement may drive the development of malignant tumors. It is clinically feasible to detect NTRK fusion: the first generation of TRK tyrosine kinase inhibitors (larotrectinib or entrectinib) can cause an unknown histological response in NTRK fusion-positive cancer patients. Entretinib is a potent and selective kinase inhibitor of the oral central nervous system that inhibits TRKA/B/C, ROS1 and ALK kinase activity[7]. NTRK been found that mutations in the multiple tumor types, including colorectal cancer, melanoma, acute myeloid leukemia and colorectal cancer[7].

NTRK3( Neurotrophic Tyrosine Kinase Receptor 3), a gene that has been found to be hypermethylated in esophageal adenocarcinoma[8],also known as TrkC, is one of three high-affinity neurotrophic factor receptors that regulate neuronal growth, differentiation and apoptosis.Rearrangements of NTRK3 and ETV6 have also been identified in congenital fibrosarcomas and secretory breast carcinomas, besides point mutations in NTRK3 have been now identified in colorectal, lung, breast, and pancreatic tumors[9]. Growing evidence suggests that NTRK3 plays an essential role in tumorigenesis, invasion and the metastatic capability of cancer cells[10]. NTRK3 is thought to play an important role in human cancers. Therefore, a better understanding of the role of NTRK3 in human cancer is an obvious first step towards making full use of it as a target for malignancies. The molecular mechanism of NTRK3 is complex and the functions of it varies in different tumors. The function and related structural variation of NTRK3 in ESCC have not been reported. Our study attempted to assess the correlation of NTRK3 gene rearrangement and gene copy number changes with ESCC prognosis by fluorescence in situ hybridization (FISH).

\section{Materials And Methods}

\section{Patients and samples}

A total of 478 ESCC patients were diagnosed and treated at Zhongshan Hospital, Fudan University during January 2007 to December 2010. None of the patients received chemotherapy or radiotherapy before surgical resection. All patients received prior written informed consent. This study was conducted in accordance with the Declaration of Helsinki and approved by the Human Research Ethics Committee of Zhongshan Hospital,Fudan University.Clinical and pathological data were collected, including gender,age,smoking,tumor location,tumor differentiation,vascular invasion, nerve invasion, and lymph node metastasis. The patients' clinicopathological features are listed in Table 1.

\section{Tissue microarray[TMA $\square$}

The TMA is constructed as described earlier[11]. In simple terms, the pathologist examines histological sections and pre-labels representative tumor areas without necrosis or hemorrhage in formalin fixed paraffin embedded (FFPE) donor blocks. 2-3 core tissues ( $2 \mathrm{~mm}$ in width and $6 \mathrm{~mm}$ in length) were taken from different representative regions of each case and arranged in receptor blocks (tissue array blocks). Our TMAs contained tumor samples, several normal esophagus, and other control tissues.

\section{NTRK3 Break Apart FISH Assay}


TMA sections(4 $\mu \mathrm{m}$ thick)were dewaxed and dehydrated》 NTRK3 rearrangement was detected by a break apart fluorescence in situ hybridization (FISH) using a commercial NTRK3 break apart FISH probe (Empire Genomics, Buffalo, NY).NTRK3 is found on chromosome 15q25.Dual-color FISH assay was performed according to Vysis/Abbott Molecular FFPE slice protocol, with some modifications.FISH analysis and signal capture of at least 50 well-defined nuclei were performed using a fluorescence microscope (BX43, Olympus, Tokyo, Japan) and a microscope digital camera (DP73, Olympus, Tokyo, Japan).NTRK3 rearrangement positive tumor cells show either an isolated 3 'signal (red only) or a separation of 5 ' and 3 'signals larger than the diameter of one signal. The NTRK3 gene copy number was calculated based on the average number of green signals in tumor cells.

\section{Statistical analysis}

Correlations and statistical analysis were performed using IBM spss 19 software. All tests were two-sided, and P<0.05 was considered statistically significant.The relationship between NTRK3 status and clinicopathological features was assessed using chi square and Fisher's exact test.The primary and secondary endpoints were cancer-related death and recurrence/metastasis. Disease-free survival (DFS) and overall survival (OS) were defined as the period from the date of surgical treatment to the date of disease progression (events: recurrence, metastasis, death) and cancer-specific survival (events: cancerrelated deaths), respectively.Correlations with overall survival were analyzed using Log Rank.The Kaplan Meier analysis with the log-rank test was used to determine the prognostic significance of DFS and OS. Kaplan Meier analysis and log-rank test Univariate analysis was conducted by $\chi 2$ test, Kappa index was used to measure the consistency of different scoring systems, and McNemar test was used to analyze statistical differences. $\mathrm{P}<0.05$ was considered as significant.

\section{Results}

\section{Clinicopathological characteristics of ESCC patients}

A total of 478 patients were enrolled in the study, and relevant clinicopathological features were shown in Table 1. In the patient group, there were 396 males (82.85\%) and 82 females (17.15\%). 184 subjects (38.49\%) were former smokers or smokers and 294 (61.51\%) were non-smokers. The lower esophagus accounted for $49.13 \%$, the middle esophagus accounted for $46.07 \%$, and the upper esophagus accounted for $4.8 \%$. The histopathological classification indicated that the tumors were poorly differentiated in $39.75 \%$, moderately differentiated in $56.69 \%$, and well differentiated in $3.56 \%$. Vascular and nerve invasion were 106 cases (22.18\%) and 165 cases (34.52\%), respectively. Lymph node metastasis was detected in 242 cases (46.3\%). According to TNM 8th edition staging, 382 cases $(79.92 \%)$ were stage I-II and 196 cases $(41.00 \%)$ were stage III-IVA.

\section{Rearrengement of NTRK3 gene in ESCC patients}

NTRK3 break-apart FISH was scored positive in 5 cases (1\%) among the 478 cases of ESCC patients.NTRK3 rearrangement was noted as a nuclear signal, and the percentage of NTRK3 rearrangement positive cells was 15\%.The correlations between NTRK3 rearrangement FISH positivity and clinical pathologic characteristics are listed in Table 1. FISH images of representative samples are shown in Figure 1. Green signals represent 5'NTRK3, and red signals as 3'NTRK3. In comparison to the 5'/3' fusion signals seen in normal cells, multiple signals for $3^{\prime}$ NTRK3 and one separated signal for 5'NTRK3 were observed in Figure $1(B)$, and a single $3^{\prime}$ NTRK3 signal pattern (loss of the signal for $5^{\prime}$ NTRK3) was observed in Figure 1 (C).Among the 478 tumors, 37 cases(7\%) were manifested with NTRK3 rearrangement and 441 cases showed negative results when $5 \%$ was defined as the cut-off value.And the NTRK3 rearrangement of $6.7 \%$ tumors was located in the range of $5 \% \leq$ NTRK3 rearrangement $<15 \%$. There was no significant correlation between NTRK3 rearrangement and gender, age, smoking, tumor size, tumor site, vascular involvement, nerve involvement, lymph node metastasis and clinical stage $(P>0.05$, Table 1$)$.

\section{Gene copy number variation of NTRK3 gene in ESCC patients}

The association between NTRK3 gene copy number variation and clinical pathologic characteristics are listed in Table 1. NTRK3 gene copy number variation FISH images of representative samples are shown in Figure 1. The group of high polysomy (CCN $\geq 4$ )was in 21 (4.4\%) cases. Low polysomy ( $3 \leq \mathrm{GCN}<4)$ was present in the other $18(3.8 \%)$ cases,and disomy in $439(91.8 \%)$ cases. There was no significant correlation between the NTRK3 gene copy number variation and sex, age, smoking, tumor site, vessel involvement, nerve involvement, and lymph node metastasis. However, NTRK3 gene copy number variation was significantly associated with tumor site $(P<0.01)$ and clinical stage $(P<0.01)$ when take $G C N<3$ as thredhold.

\section{Correlation between NTRK3 rearrangement and copy number variation}

NTRK3 rearrangement or copy number variation are analyzed and showed in Table2. NTRK3 rearrangement FISH positive showed 1 case in GCN $\geq 4$ and 4 cases in GCN $<3$. The patients with NTRK3 rearrangement located in the range of $5 \% \leq$ NTRK3rearrangement $<15 \%$ was 4 case in GCN $\geq 4,4$ cases in $3 \leq \mathrm{GCN}<4$ and 4 cases in $\mathrm{GCN}<3$. Among patients with NTRK3 rearrangement $<5 \%, 16$ cases was in $\mathrm{GCN} \geq 4,14$ cases in $3 \leq \mathrm{GCN}<4$ and 411 cases in $\mathrm{GCN}<3$. The difference between NTRK3 rearrangement and copy number variation had statistical significance $(P=0.003)$, which is shown in Table 2.

\section{Survival analysis of ESCC patients}

To determine whether NTRK3 rearrangement or copy number variation is a prognostic factor in ESCC, we performed a Kaplan Meier survival analysis. The different levels of NTRK3 rearrangement or copy number variation were compared using a log-rank test.252 patients (52.72\%) had died from esophageal cancer and 226 patients (47.28\%) had disease progression during the follow up.As was shown in Figure 2, there was no significant difference in survival between NTRK3 rearrangement and the DFS and OS of ESCC when NTRK3 rearrangement located in the range of $5 \% \leq$ NTRK3rearrangement $<15 \%$ (DFS, $\mathrm{P}=0.767$; OS, $\mathrm{P}=0.926$ )(Figure $2 \mathrm{~B}, \mathrm{E}$ ).At the threshold of $15 \%$, NTRK3 rearranged groups had worse DFS and OS than the wild type groups, but no significant difference was observed(15\%, DFS, $P=0.601 ; O S, P=0.551)$ (Figure 2 C,F). 
Besides,Comparing with group of GCN $<3$, the high NTRK3 gene copy number groups was significantly associated with worse patient survival ( GCN $\geq 4$ DFS, $P=0.213$; OS, $P=0.165$ )(Figure $3 \mathrm{C}, F$ ), although this finding did not reach statistical significance in DFS or in OS. In order to further determine the role of NTRK3 in the prognosis of patients with esophageal cancer, we performed a Kaplan Meier survival analysis on the I+II phase patiens and III+1Va phase patiens respectively. Furthermore, it was found that in clinical stage I+II patients, the DFS and OS of patients of high NTRK3 gene copy number group(GCN $\geq 4$ as the boundary value), were significantly longer (DFS, $P=0.120$; OS, $P=0.095$ )(Figure $3 \mathrm{I}, \mathrm{L}$ ). In patients with stage III+IVa, there was no significant difference in $D F S$ and OS between patients of high NTRK3 gene copy number group (GCN $\geq 4$ )(DFS, $P=0.376$; OS, $P=0.319$ ) (Fgure4 O,R)with the other group.

\section{Discussion}

The NTRK fusion was first discovered in colon cancer in 1986 when a translocation of tpm3-NTRK1 was detected in a tumor biopsy[12]. Several NTRK gene fusion have been identified as potential tumorigenic sites that coexist in many tumors. For example, etv6-NTRK3 gene fusion is present in $93 \%$ of breast cancers and $100 \%$ of breast mimic cancers (MASC)[13]. Despite the plethora of reports of gene fusions involving NTRK3 in various malignancies including Glioblastoma, Fibrosarcoma, AML, GIST, Colorectal[9, 14-17],there are relatively few studies investigating NTRK3 rearrangement in ESCC. Furthermore, there are no studies simultaneously addressing both NTRK3 rearrangement and amplification in esophageal squamous cell carcinoma.

We have shown that NTRK3 rearrangement is a rare event in ESCC (5 cases (1\%)among the 478 cases of ESCC) in our study.There was no significant correlation between the NTRK3 rearrangement and gender, age, smoking, tumor size, tumor site, vascular involvement, nerve involvement, lymph node metastasis and clinical stage. The result of Kaplan Meier survival analysis also show that there is no relevance in our opinion to search for NTRK3 rearrangement in the prognosis of patients with esophageal cancer.lt may due to low frequency of NTRK3 rearrangement.

Furthermore, amplification of the NTRK3 seems to be another possible mechanism of genetic alteration in ESCC. Though there was also no significant correlation between the NTRK3 gene copy number variation and the clinicopathological parameters. Howerver,the high NTRK3 gene copy number group was significantly associated with worse patient survival ( $G C N \geq 4)$, although this finding did not reach statistical significance in DFS or in OS.To further confirm the role of amplification of the NTRK3 in the prognosis of patients with esophageal cancer, Kaplan Meier survival analysis was caculated in the I+II phase and III+IVa phase respectively. Furthermore, it was found that in clinical stage I+II patients, high NTRK3 gene copy number group(GCN $\geq 4$ ) was correlated with bad prognosis. In clinical stage III+IVa patients, there was no significant correlation between the amplification of the NTRK3 and the survival of patients.

Our results demonstrated high NTRK3 gene copy number has an impact on prognosis in early TNM stage (stage I+II) instead in advanced TNM stage (stage III+IVa) . And there was no prognostic difference between stage I+II ESCC with low NTRK3 gene copy number and stage III+IVa ESCC.

This indicates that the prognostic significance of amplification of the NTRK3 is conditional and limited to the early clinical stage of ESCC development. These results suggest that the prognostic significance of amplification of the NTRK3 is limited. The complex molecular mechanism in late ESCC may affect the biological effect of NTRK3. These data also indicate that in clinical practice, the prognostic significance of molecular markers should be evaluated in different tumor stages. To the best of our knowledge, this is the first report correlating NTRK3 with clinicopathological parameters or prognosis of ESCC.

Studies on the correlation between NTRK3 copy number changes and esophageal cancer indicate that NTRK3 has an impact on prognosis in early TNM stage (stage I-II). It was found that in early ESCC, increased NTRK3 copy number (critical value 3 ) was an independent prognostic factor, suggesting a better prognosis for patients. In patients with advanced ESCC, NTRK3 copy number increased without prognostic significance. It is suggested that the prognostic significance of increased NTRK3 copy number is limited. The complex molecular mechanism in late ESCC may affect the biological function of NTRK3. These data also indicate that in clinical practice, the prognostic significance of molecular markers should be evaluated in different stages of tumors. The research still has some limitations. To assess the function of NTRK3 in esophageal cancer populations, these samples may be further studied in subsequent work. The specific abnormalities of NTRK3 in esophageal cancer (mutation, CNVs, methylation) and the transcriptional status of mRNA/protein levels remain to be further studied, which will provide more information on the role of NKRK3 in esophageal cancer.

\section{Declarations}

\section{Authors' contributions}

Huijuan Xu and Dongxian Jiang contributed equally to this work.Yingyong Hou囚the corresponding author, performed study concept and design.Huijuan Xu and Dongxian Jiang performed development of methodology, acquisition, analysis, interpretation of data, statistical analysis, writing, review and revision of the paper.Fuhan Zhang®Zixiang Yu囚Jie Huang®Akesu Sujie囚Jianfang Xu provided technical and material support.All authors reviewed the manuscript.

\section{Competing interests}

The authors claim that they have no competing interests.

\section{Funds}

This research was supported by the Science and Technology Program of Xiamen(3502Z20184003)and the incubation project of Xiamen Branch of Zhongshan Hospital,Fudan University(2020ZSXMYJ01).

\section{Ethics approval and consent to participate}

This research was approved by the Ethics Committee of Zhongshan Hospital of Fudan University (Shanghai, China).Informed consent Written informed consent was obtained from patients for use of their surgical specimens for research purposes 


\section{Data availability statement}

The corresponding author can provide the data of this study if reasonable.

\section{References}

1. Zhao P, Dai M, Chen W, Li N: Cancer trends in China. Jpn J Clin Oncol 2010, 40(4):281-285.

2. Chen W, Zheng R, Baade PD, Zhang S, Zeng H, Bray F, Jemal A, Yu XQ, He J: Cancer statistics in China, 2015. CA Cancer J Clin 2016, 66(2):115-132.

3. Abnet CC, Arnold M, Wei WQ: Epidemiology of Esophageal Squamous Cell Carcinoma. Gastroenterology 2018, 154(2):360-373.

4. Ando N, Ozawa S, Kitagawa Y, Shinozawa Y, Kitajima M: Improvement in the results of surgical treatment of advanced squamous esophageal carcinoma during 15 consecutive years. Ann Surg 2000, 232(2):225-232.

5. Lin DC, Hao JJ, Nagata Y, Xu L, Shang L, Meng X, Sato Y, Okuno Y, Varela AM, Ding LW et al: Genomic and molecular characterization of esophageal squamous cell carcinoma. Nat Genet 2014, 46(5):467-473.

6. Lange AM, Lo HW: Inhibiting TRK Proteins in Clinical Cancer Therapy. Cancers (Basel) 2018, 10(4).

7. Cocco E, Scaltriti M, Drilon A: NTRK fusion-positive cancers and TRK inhibitor therapy. Nat Rev Clin Oncol 2018, 15(12):731-747.

8. Kaz AM, Wong CJ, Luo Y, Virgin JB, Washington MK, Willis JE, Leidner RS, Chak A, Grady WM: DNA methylation profiling in Barrett's esophagus and esophageal adenocarcinoma reveals unique methylation signatures and molecular subclasses. Epigenetics 2011, 6(12):1403-1412.

9. Hechtman JF, Benayed R, Hyman DM, Drilon A, Zehir A, Frosina D, Arcila ME, Dogan S, Klimstra DS, Ladanyi M et al: Pan-Trk Immunohistochemistry Is an Efficient and Reliable Screen for the Detection of NTRK Fusions. Am J Surg Pathol 2017, 41(11):1547-1551.

10. Luo Y, Kaz AM, Kanngurn S, Welsch P, Morris SM, Wang J, Lutterbaugh JD, Markowitz SD, Grady WM: NTRK3 is a potential tumor suppressor gene commonly inactivated by epigenetic mechanisms in colorectal cancer. PLoS Genet 2013, 9(7):e1003552.

11. Shi Y, He D, Hou Y, Hu Q, Xu C, Liu Y, Jiang D, Su J, Zeng H, Tan Y: An alternative high output tissue microarray technique. Diagn Pathol $2013,8: 9$.

12. Martin-Zanca D, Hughes SH, Barbacid M: A human oncogene formed by the fusion of truncated tropomyosin and protein tyrosine kinase sequences. Nature 1986, 319(6056):743-748.

13. Vaishnavi A, Le AT, Doebele RC: TRKing down an old oncogene in a new era of targeted therapy. Cancer Discov 2015, 5(1):25-34.

14. Shah N, Lankerovich M, Lee H, Yoon JG, Schroeder B, Foltz G: Exploration of the gene fusion landscape of glioblastoma using transcriptome sequencing and copy number data. BMC Genomics 2013, $14: 818$.

15. Knezevich SR, McFadden DE, Tao W, Lim JF, Sorensen PH: A novel ETV6-NTRK3 gene fusion in congenital fibrosarcoma. Nat Genet 1998, 18(2):184-187.

16. Smith KM, Fagan PC, Pomari E, Germano G, Frasson C, Walsh C, Silverman I, Bonvini P, Li G: Antitumor Activity of Entrectinib, a Pan-TRK, ROS1, and ALK Inhibitor, in ETV6-NTRK3-Positive Acute Myeloid Leukemia. Mol Cancer Ther 2018, 17(2):455-463.

17. Brenca M, Rossi S, Polano M, Gasparotto D, Zanatta L, Racanelli D, Valori L, Lamon S, Dei Tos AP, Maestro R: Transcriptome sequencing identifies ETV6NTRK3 as a gene fusion involved in GIST. J Pathol 2016, 238(4):543-549.

\section{Tables}

Table 1: Correlation between clinicopathological parameters and NTRK3 gene rearrangement and copy number variation in ESCC 


\section{NTRK3 rearrangement}

All patients

Characteristics
$5 \% \leq$ NTRK3

rearrangement $\geq 15 \%$

rearrangement $<15 \%$
NTRK3 gene copy number (GCN)

$\mathrm{GCN} \geq 4 \quad 3 \leq \mathrm{GCN}<4$

GC

$\begin{array}{lllllllllllllllll}\text { No. } & \text { Yes } & \text { No } & \text { P- } & \text { Yes } & \text { No } & P & \text { Yes } & \text { No } & \text { P- } & \text { Yes } & \text { No } & \text { P- } & \text { Yes } & \text { No } & P & \text { Yes }\end{array}$ value Yes No $P$ value ves No

Sex

0.205

0.365

0.5

Male

$396(82.85 \%) \quad 3 \quad 393$

$\begin{array}{lllll}29 & 367 & 0.167 & 364 & 32\end{array}$

$17 \quad 379$

Female $82(17.15 \%) \quad 2 \quad 80$

Age

0.348

$3 \quad 79$

$\geq 60$

274(57.32\%) $2 \quad 282$

$<60$

204(42.68\%) $3 \quad 201$

Smoking

0.634

Yes

No

184(38.49\%) $2 \quad 181$

292

294(61.51\%)

(1)

Tumor size

II

Tumor site

208(43.51\%) $1 \quad 207$

0.278

0.215

$\sqrt{2}+x^{2}$

0.147

$4 \quad 78$

$16 \quad 268$

26618

0.147

0.449

$10 \quad 264$

0.234

$\begin{array}{llll}16 & 380 & 0.377 & 36\end{array}$

$16 \quad 188$

0.458

$168 \quad 15$

0.449

$273 \quad 22$

$10 \quad 284$

0.279

0.443

$191 \quad 17$

0.443

$270(56.49 \%) \quad 4 \quad 266$

$16 \quad 254$

$250 \quad 20$

$14 \quad 256$

0.779

0.552

Upper

22(4.80\%) 0

0.495

$1 \quad 21$

17

21

1

11

$11 \quad 214$

Middle

211(46.07\%) $3 \quad 208$

$16 \quad 195$

19219

9202

202

Low 225(49.13\%) $1 \quad 224$

Differentiation

Poor

190(39.75\%) 1189

0.508

0.889

0.903

$0 \quad 22$

93

2

$80 \quad 76$

Moderate

271(56.69\%) $4 \quad 267$

$12 \quad 178$

$177+13$

$\begin{array}{llll}177 & 13 & 9 & 181\end{array}$

$\begin{array}{llll}247 & 24 & 12 & 259\end{array}$

0.57

\begin{tabular}{rrrrr} 
& 11 & 263 & & $25 i$ \\
& 7 & 197 & & 18 \\
\hline .134 & & & 0.577 & \\
\hline & 7 & 177 & & $16 t$
\end{tabular}

Well

$17(3.56 \%) \quad 0 \quad 17$

Vessel

involvement

\begin{tabular}{|c|c|c|c|c|c|c|c|c|c|c|c|c|c|}
\hline Yes & $106(22.18 \%)$ & 1 & 105 & 7 & 99 & & 98 & 8 & 6 & 100 & 4 & 102 & 95 \\
\hline No & $372(77.82 \%)$ & 4 & 368 & 25 & 347 & & 343 & 29 & 15 & 357 & 14 & 358 & 34 \\
\hline $\begin{array}{l}\text { Nerve } \\
\text { involvement }\end{array}$ & & & & & & 0.164 & & & & & & & \\
\hline Yes & $165(34.52 \%)$ & 1 & 164 & 8 & 157 & & 156 & 9 & 7 & 158 & 5 & 160 & 15 \\
\hline No & $313(65.48 \%)$ & 4 & 309 & 24 & 289 & & 285 & 28 & 14 & 299 & 13 & 300 & 286 \\
\hline $\begin{array}{l}\text { Lymph node } \\
\text { metastasis }\end{array}$ & & & & & & 0.456 & & & & & & & \\
\hline Yes & $228(47.70 \%)$ & 4 & 224 & 16 & 212 & & 208 & 20 & 10 & 218 & 7 & 221 & 211 \\
\hline No & $250(52.30 \%)$ & 1 & 249 & 16 & 234 & & 233 & 17 & 11 & 239 & 11 & 239 & $22 \varepsilon$ \\
\hline Clinical stage & & & & & & 0.44 & & & & & & & \\
\hline III-Iva & $196(41.00 \%)$ & 3 & 193 & 14 & 182 & & 179 & 17 & 7 & 189 & 4 & 192 & 18 ? \\
\hline $\mathrm{H}$ & $282(79.92 \%)$ & 2 & 280 & 18 & 264 & & 262 & 20 & 14 & 368 & 14 & 368 & 25 \\
\hline Death of EC & & & & & & 0.450 & & & & & & & \\
\hline Yes & $252(52.72 \%)$ & 2 & 249 & 16 & 235 & & 233 & 18 & 14 & 238 & 6 & 246 & $23:$ \\
\hline No & $226(47.28 \%)$ & 3 & 223 & 16 & 210 & & 207 & 19 & 7 & 219 & 12 & 214 & 20 \\
\hline
\end{tabular}

Table 2: Number of cases that simultaneously with NTRK3 rearrangement and copy number variation 


\begin{tabular}{|c|c|c|c|c|c|}
\hline \multicolumn{2}{|c|}{ NTRK3 rearrangement } & \multicolumn{4}{|c|}{ NTRK3 gene copy number (GCN) } \\
\hline & & $\mathrm{GCN} \geq 4$ & $3 \leq \mathrm{GCN}<4$ & $\mathrm{GCN}<3$ & P Value \\
\hline$<5 \%$ & 441 & 16 & 14 & 411 & 0.003 \\
\hline $5 \%-15 \%$ & 32 & 4 & 4 & 24 & \\
\hline$\geq 15 \%$ & 5 & 1 & 0 & 4 & \\
\hline
\end{tabular}

\section{Figures}
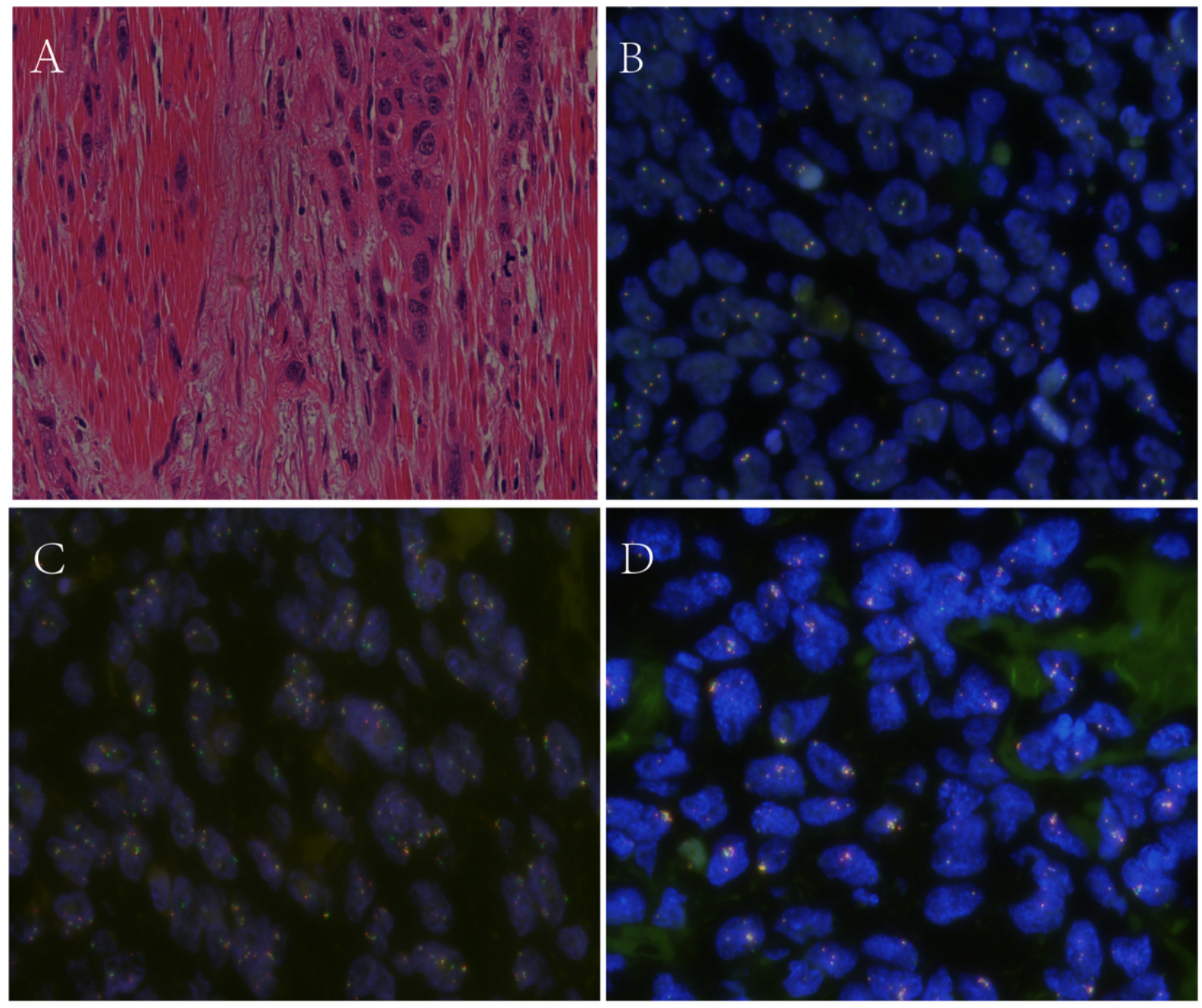

\section{Figure 1}

Fluorescent in situ hybridization detection of NTRK3 gene variation. A,HE; B,images without gene rearrangement; C,gene rearrangement(Blue: interphase nuclei stained with DAPI; Red: 15q25.3 labeled with Cy3; green: centrosome of 15 chromosome labeling with SpG.).D, Nucleus with cluster-like amplification. 

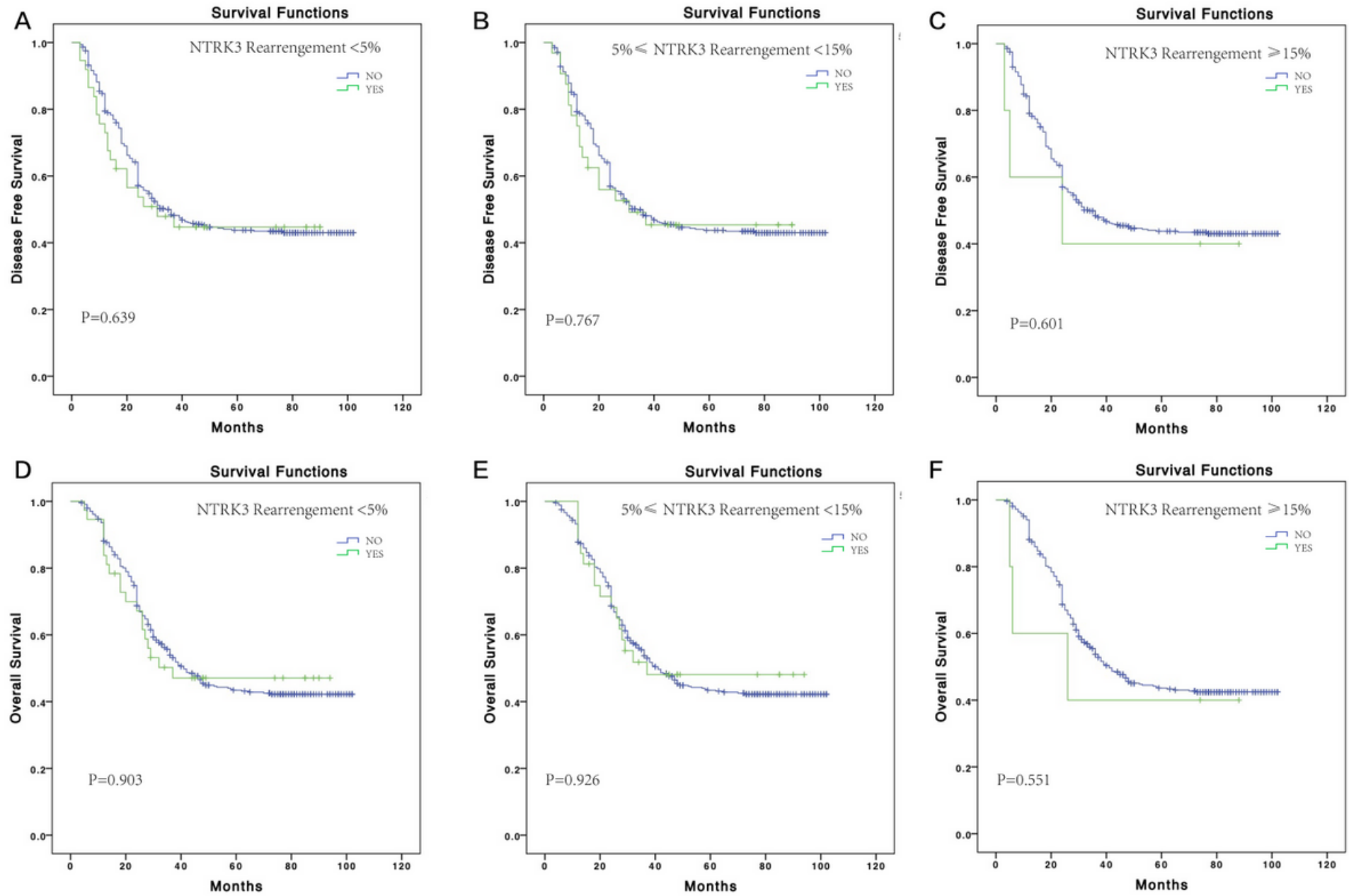

Figure 2

Correlaction between NTRK3 rearrangement and survival in ESCC.Among all esophageal squamous cell carcinoma patients,the DFS(C) and OS(F) were potentially worse in positive patients with NTRK3 rearrangement $\geq 15 \%$ than in negative patients $(P=0.601$ and 0.531$)$. NTRK3rearrangement positive patients is less than $15 \%$ with negative the prognosis of patients with no statistically significant difference $(5 \%$ for the boundary value, $\mathrm{P}=0.639$ and $0.903 ; 5 \%-15 \%$ for the boundary value, $P=0.767$ and 0.926 ). 


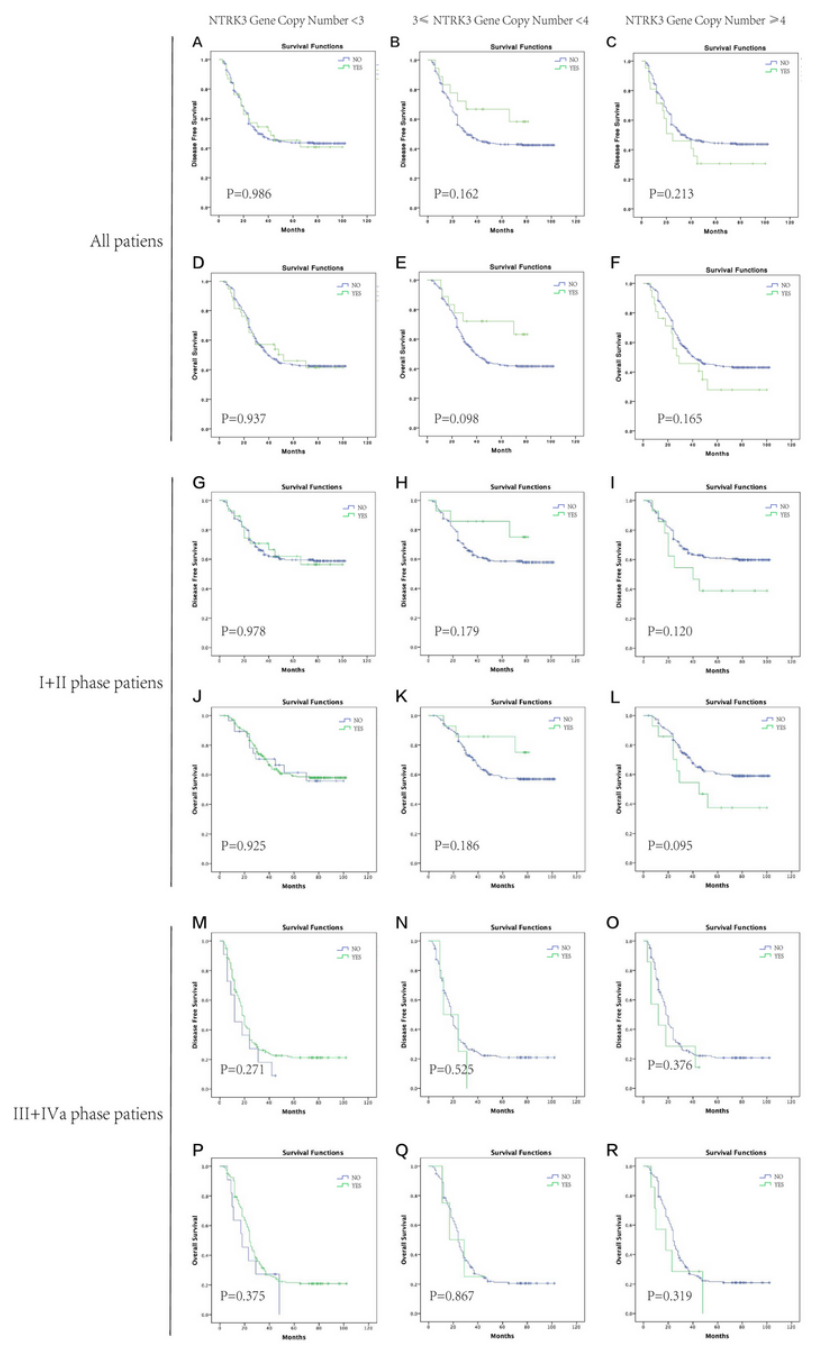

\section{Figure 3}

Correlaction between NTRK3 gene copy number variation and survival in ESCC.A-F,among all esophageal squamous cell carcinoma patients, the DFS and OS of positive patients with NTRK3 Copy Number Variation $\geq 3$ were potentially better than those of negative patients $(3 \leq N T R K 3$ GCN $<4$ DFS, $P=0.0 .162$; OS, $P=$ 0.098; NTRK3 GCN $\geq 4$ DFS, P = 0.213; OS, P = 0.165); There were no significant differences of the DFS and OS between positive patients with NTRK3 Copy Number Variation $<3$ and negative patients (NTRK3 GCN<3 DFS, P=0.986; OS, P = 0.937).G-L,in clinical stage I+II patients, the DFS and OS of patients of high NTRK3 gene copy number group(GCN $\geq 4$ as the boundary value), were significantly longer (DFS, $P=0.120 ; 0 S, P=0.095)$. M-R,in patients with stage III+IVa, there was no significant difference in DFS and OS between patients of high NTRK3 gene copy number group $(G C N \geq 4)(D F S, P=0.376 ; 0 S, P=0.319)$ with the other group. 